\title{
Ductile failure analysis of high strength steel in hot forming based on micromechanical damage model
}

\author{
Liang Ying ${ }^{1}$, Wenquan $\mathrm{Liu}^{2}$, Dantong Wang ${ }^{1}$ and Ping $\mathrm{Hu}^{1, a}$ \\ ${ }^{1}$ School of Automotive Engineering, Dalian University of Technology, 116024 Dalian Liaoning, China \\ ${ }^{2}$ Department of Engineering Mechanics, Dalian University of Technology, 116024 Dalian Liaoning, China
}

\begin{abstract}
The damage evolution of high strength steel at elevated temperature is investigated by using the Gurson-Tvergaard-Needleman (GTN) model. A hybrid method integrated thermal tensile test and numerical technique is employed to identify the damage parameters. The analysis results show that the damage parameters are different at different temperature as the variation of tested material microstructure. Furthermore, the calibrated damage parameters are implemented to simulate a bugling forming at elevated temperature. The experimental results show the availability of GTN damage model in analyzing sheet formability in hot forming.
\end{abstract}

\section{Introduction}

High strength steel (HHS) components have been widely used for the purpose to reduce the weight of automobile and guarantee crash safety as well. Nevertheless, a major drawback of the HHS is the low formability and severe spring-back at ambient temperature. Hot forming of high strength steel, especially $22 \mathrm{MnB} 5$, is an innovative technology which integrates the hot forging and cold forming, so that it can solve these defects quite well[1]. However, the elastic modulus and tensile strength decrease markedly and the frictional coefficient increases significantly with the increase of temperature. Therefore, forming fracture is also a major problem to study during hot forming process.

The efficient method for predicting material formability is based on the damage mechanics theory. The microscopic damage analysis proposed by McClintock[2], developed by Gurson[3] and lots of later authors, has been widely used to investigate the fracture behavior of metal sheet. GTN model is one of the most widely used damage models for sheet forming. However, there are few reports on the damage investigation of high strength steels based on GTN model for hot forming process. Klocker et al[ [4] have used an axisymmetric unit cell model to study the void growth and coalescence in metals deformed at elevated temperature. And the critical void volume fraction $f_{c}$ shows strong dependency upon temperature. Moreover, the similar results were discovered by Tinet et al[5] experimentally, but the failure void fraction $f_{F}$ nearly kept nearly in constant. Moreover, the microstructure of boron steel 22MnB5 will vary with the variation of temperature. Thus, the investigation of damage evolution for hot forming process must fully consider the influence of temperature.

In this work, the ductile damage of $22 \mathrm{MnB} 5$ at elevated temperature has been studied. The damage parameters of GTN model are optimized by a hybrid method integrated thermal tensile test and numerical technique for different tested temperature. Eventually, the damage model with the calibrated parameters are implemented to simulate a high temperature bugling process by means of the finite element code ABAQUS. The corresponding experiment is also conducted and the comparison of simulation data and experiment results has been carried out.

\section{Material damage model}

\subsection{GTN damage model}

The damage model proposed by Gurson and modified by Tvergaard and Needleman[6] known as GTN model, which has the expression:

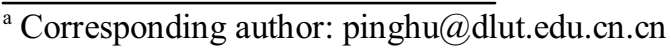




$$
\begin{gathered}
\varphi\left(\sigma_{e q}, \sigma_{h}, f^{*}\right)=\left(\frac{\sigma_{e q}}{\bar{\sigma}_{y}}\right)+2 f^{*} q_{1} \cosh \left(-\frac{3 q_{2} \sigma_{h}}{2 \bar{\sigma}_{y}}\right)- \\
\left(1+q_{3} f^{* 2}\right)=0
\end{gathered}
$$

Where $\sigma_{e q}$ is the macro equivalent stress of Cauchy stress tensor, $\sigma_{h}$ is the macro hydrostatic stress, $\bar{\sigma}_{y}$ is the flow stress of matrix material, the parameters $q_{1}, q_{2}, q_{3}$ are introduced by Tvergaard[7] to improve the prediction accuracy and the suggestive value for metal have been adopted, $q_{1}=1.5, q_{2}=1.0, q_{3}=q_{1}^{2}$. Here, $f^{*}$ is the function of damage volume fraction $f$ :

$$
f^{*}= \begin{cases}f & f \leq f_{c} \\ f_{c}+\left(f-f_{c}\right) \frac{f_{u}-f_{c}}{f_{F}-f_{c}} & f_{c}<f \leq f_{F} \\ 1 / q_{1} & f \geq f_{F}\end{cases}
$$

Where $f_{c}$ is the critical void volume fraction at which void coalescence starts, $f_{u}=1 / q_{1}$ is the ultimate value of $f^{*}$ at material failure, $f_{F}$ is the final void volume fraction at material fracture. It can be seen when $f_{c}<f \leq f_{F}$, there is an acceleration of $f^{*}$. It was introduced by Tvergaard and Needleman to characterize the damage accelerated evolution process caused by void coalescence.

In general, the damage evolution of metal materials during plastic deformation includes two parts, the growth and nucleation of voids as shown in Eq. (3), the detailed descriptions can be found in paper[8]:

$$
\dot{f}=\dot{f}_{\text {growth }}+\dot{f}_{\text {nucleation }}
$$

The equivalent plastic strain $d \bar{\varepsilon}^{p l}$ of matrix material can be determined by the equivalent plastic work rule:

$$
(1-f) \bar{\sigma}_{y} d \bar{\varepsilon}^{p l}=\boldsymbol{\sigma} d \varepsilon^{p l}
$$

Where $\boldsymbol{\sigma}$ and $\varepsilon^{p l}$ are the macro plastic strain tensor and macro stress tensor, respectively.

For hot forming process, the microstructure and mechanical property of high strength steel are different with the variation of temperature. Therefore, to investigate the damage evolution behavior under hot stamping process, the damage parameters under various temperatures should be determined.

\subsection{The hardening law of matrix material}

The flow behavior of matrix material $22 \mathrm{MnB} 5$ at elevated temperature is characterized by the modified Norton-Hoff hardening law:

$$
\sigma=K \cdot \exp \left(\frac{\beta}{T}\right) \cdot\left(\varepsilon_{0}+\varepsilon\right)^{n(T)} \cdot \dot{\varepsilon}^{m(T)}
$$

with

$$
\begin{array}{r}
n(T)=n_{0} \exp \left(-c_{n}\left(T_{i}-T_{0}\right)\right) \\
m(T)=m_{0} \exp \left(c_{m}\left(T_{i}-T_{0}\right)\right)
\end{array}
$$

where $K, \beta, \varepsilon_{0}, n_{0}, m_{0}, \mathrm{c}_{n}, c_{m}$ are material constants and $T_{0}$ is the reference temperature. For tested material at different temperature, the material constants are determined from the thermal tensile test in next section. The determined constants are listed in Table1. It can be seen that the effect of temperature on hardening exponent is vanished for matrix-material without damage. The method to obtain material mechanical property without damage can be found in literature[9].

TABLE1. Norton-Hoff constants for 22MnB5 at elevated temperature

\begin{tabular}{ccccccc}
\hline$K$ & $\beta$ & $\varepsilon_{0}$ & $n_{0}$ & $m_{0}$ & $\mathrm{c}_{n}$ & $c_{m}$ \\
\hline 26.647 & 2500 & 0.007389 & 0.2653 & 0.0101 & 0.0 & 0.000828 \\
\hline
\end{tabular}

\section{Hot tensile test and parameter calibration}

\subsection{Hot tensile test}

According to actual hot forming process, the temperature-time procedure of hot tensile test with a strain rate $0.1 \mathrm{~s}^{-1}$ is executed as shown in Fig 1 . The experimental material is hot rolled boron steel $22 \mathrm{MnB} 5$ with a thickness of $2 \mathrm{~mm}$. The dog bone shaped samples with gauge length $50 \mathrm{~mm}$ and width $20 \mathrm{~mm}$ are used in the test.

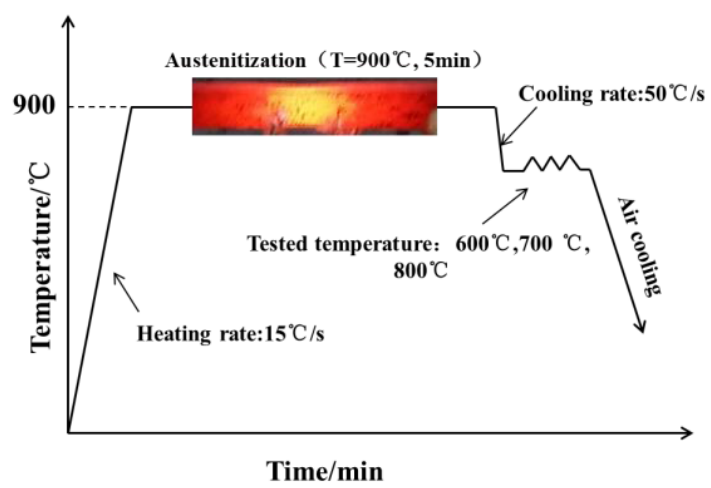

Figure 1. Procedure of hot tensile test

The symbols in Fig 2(a) represent the true stress-strain relation of boron steel $22 \mathrm{MnB} 5$ at $600^{\circ} \mathrm{C}$, $700{ }^{\circ} \mathrm{C}$ and $800{ }^{\circ} \mathrm{C}$. It can be seen that deformation temperature has a strong influence on tensile force. The peak stress decreases with the increase of temperature markedly. For each test temperature, the flow stress firstly increases due to work hardening. After reaching peak stress, the flow curve begins to drop until the occurrence of final fracture as the domination of voids damage. The stress-plastic strain curves without damage for different tested temperature predicted by Norton-Hoff model are shown in Fig 2(b) which are 
consistent with the experimental results.

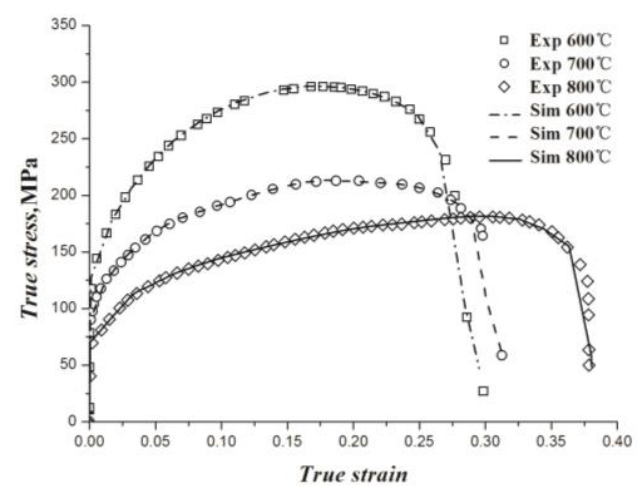

(a) comparsion of experimental (symbol) and predicted (solid) stress-strain curves of whole process

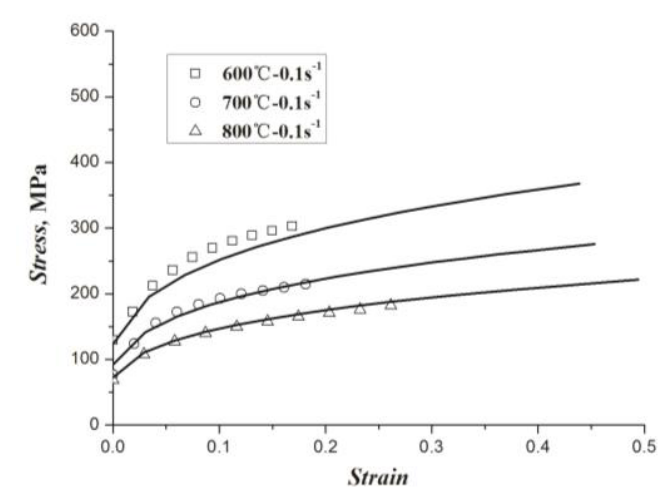

(b) comparsion of experimental (symbol) and fitted (solid) stress-strain relationships of hardening phase

Figure 2. Comparsion of experimental and predicted curves

\subsection{Calibration of damage parameters}

In general, the GTN model has 9 parameters as mentioned above. To minimize the calibration procedure for these parameters, $q_{0}, q_{1}, q_{3}, \varepsilon_{N}, S$ have been kept constants as identified in section2.

Hence, $f_{0}, f_{N}, f_{c}, f_{F}$ are the final damage parameters needed to be determined for boron steel $22 \mathrm{MnB} 5$ at different temperature.

To calibrate the damage parameter, a hybrid method integrated experiment and numerical technique is employed. The thermal tensile simulations at various temperatures are conducted as the hot tensile test mentioned above. Naturally, the simulation curve and experimental one can match each other very well if the peak point and fracture point from these two ways match each other, respectively.

The procedure of this hybrid method to identity these four damage parameters can be summarized as follows:

a. Obtaining the experimental stress-strain data at different temperatures.

b. Simulation design for tensile test by central composite experimental design

c. Extracting response variables, peak point and fracture point, for each simulation. d. Establishing response surface models for peak stress, peak strain, fracture stress and fracture strain.

e. Calculating damage parameters by Genetic Algorithm method. The GA program is realized through MATLAB software.

f. Using the optimum value of damage parameters to simulate tensile test and compare with experimental results.

The optimal value of damage parameters are listed in Table 2 and the predicted stress-strain data (solid curves) using these damage parameters are shown in Fig. 2(a), which show a good accordance with experimental results. From Table 2, we can know that the damage parameters vary with the variation of tested temperature which can be explained by the microstructure transformation of boron steel 22MnB5 at different temperatures.

Table2. Damage parameters for 22MnB5 at different temperatures

\begin{tabular}{ccccc}
\hline Temperature & $f_{0}$ & $f_{N}$ & $f_{c}$ & $f_{F}$ \\
\hline $600^{\circ} \mathrm{C}$ & 0.0005 & 0.0110 & 0.0401 & 0.1345 \\
$700^{\circ} \mathrm{C}$ & 0.0007 & 0.0141 & 0.0495 & 0.1410 \\
$800^{\circ} \mathrm{C}$ & 0.0008 & 0.0165 & 0.0592 & 0.1550 \\
\hline
\end{tabular}

\section{Numerical simulation of bulging experiment}

To verify the calibrated damage parameters, a bugling process at elevated temperature is simulated and the corresponding experiment is also carried out.

\subsection{Bugling apparatus and experiment process}

To investigate the formability of metal sheet at elevated temperature, a bugling apparatus is designed and developed as shown in Fig 4. The device consists a hemispherical punch, a blank holder and a die with draw-bead. This device can be heated up to $900^{\circ} \mathrm{C}$ and the temperature of tested blank is kept during forming process.

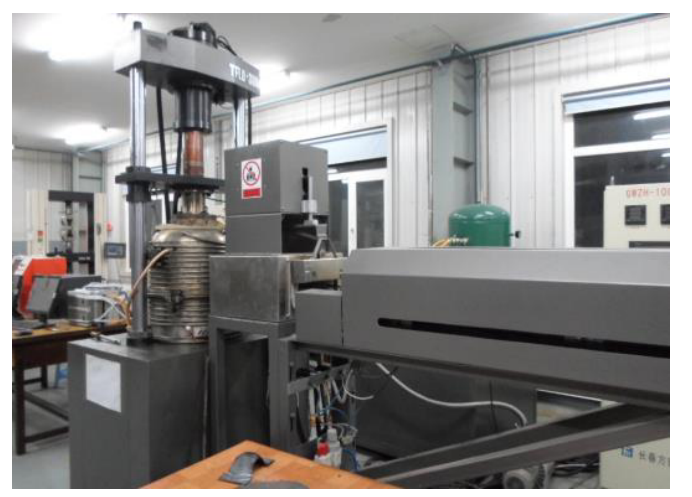

Figure 3. Experiment apparatus for high temperature bugling

In this work, the bugling experiment at $700^{\circ} \mathrm{C}$ has been carried out to identify the effectiveness of above damage parameters. The same procedure as hot tensile 
test was adopted: firstly, the metal sheet is heated to $900^{\circ} \mathrm{C}$ in furnace and this temperature is preserved $5 \mathrm{~min}$ for soak austenitization. Then the sheet metal blank is rapidly transferred into the bulging apparatus and rapidly cooled to the tested temperature with an average cooling rate $50^{\circ} \mathrm{C} / \mathrm{s}$ and deformed at this temperature until failure. Meanwhile, the tools are also heated up to tested temperature and remained all the process. The punch velocity is set to $500 \mathrm{~mm} / \mathrm{min}$ and a blank holder force of $100 \mathrm{kN}$ is provided by the upper die. The mixture of graphite and molybdenum disulfide is laid on the punch surface before bugling to reduce friction between the sheet metal specimen and the punch.

\subsection{Numerical simulation for bugling test}

Consistent with the experiment apparatus, the finite element model is established as shown in Fig 4. Both the metal blank and tools are meshed using quadrilateral shell element but the tools are set as coupled rigid bodies to avoid deformation. Because of the constant temperature condition during whole bugling process, the thermal coefficient is not needed. The hardening relation predicted by modified Norton-Hoff and material damage parameters at $700^{\circ} \mathrm{C}$ obtained above are implemented in the simulation model. The friction coefficient is set to 0.22 which is less than the common value 0.4 in hot forming as the addition of lubricant. And the sample is a $180 * 180 \mathrm{~mm}$ square blank with a thickness of $2 \mathrm{~mm}$.

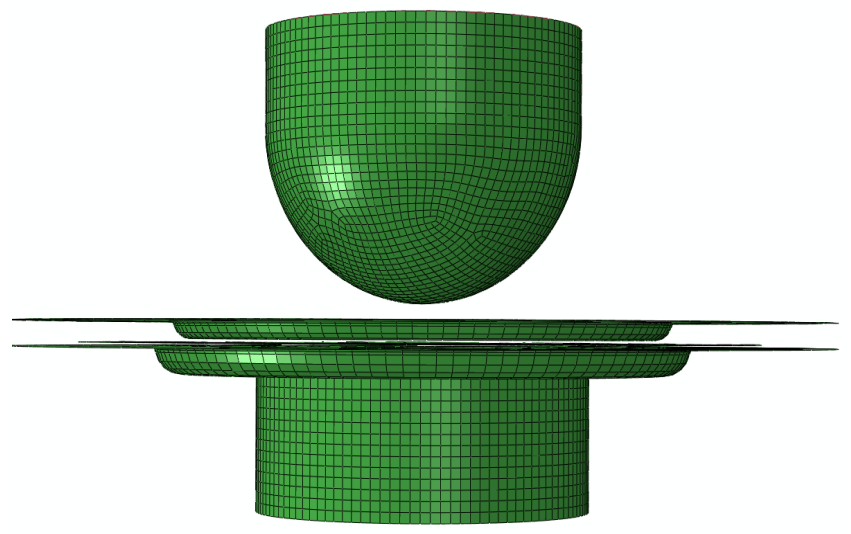

Figure 4. Simulation model of bugling forming

Fig 5 shows the comparison between the experimental and numerical punch force-stroke curves. The solid curve is the experimental result; the symbol point is the predicted result with material damage while the dotted line is the predicted result without damage. It can be seen that the curve predicted with damage can match the experimental curve very well which proves the availability of GTN model and the calibrated damage parameter. One more thing should be noted is that the initial simulation curve has some fluctuation, the reason for this probably causing by the instability of contact algorithm. So some work to smooth the curves has been done but the effectiveness is still maintained.

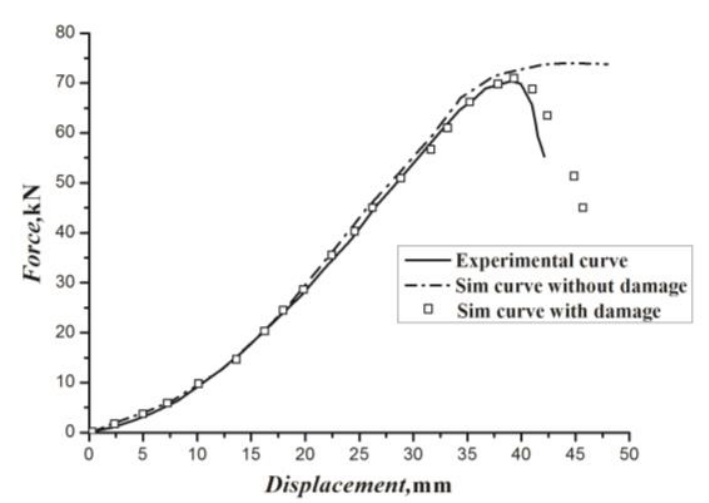

Figure 5. Comparison between the experimental and numerical punch force-stroke curves

Fig 6 reports the void damage distribution of tested sample at different deformation phases. It shows that damage mainly distributes at the location where the blank contacts the punch and draw bead firstly. Along with the downward of punch, void damage begins to gather near the dome of the blank which is accordance with the experimental result as shown in Fig 7.

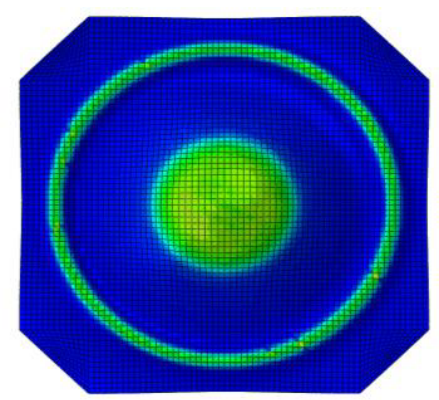

(a) Initial period of bugling

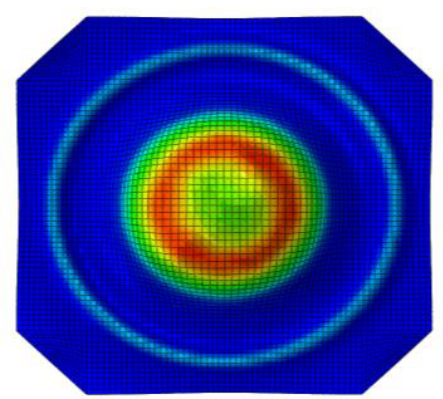

(b) Deformation phase before fracture

Figure 6. Numerical void damage distribution at different bugling phases

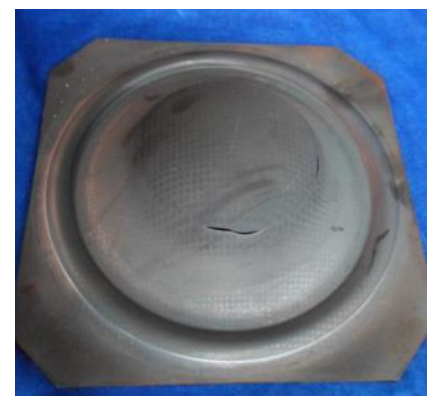

Figure 7. Sample after bugling forming at $700^{\circ} \mathrm{C}$ 
To study the damage evolution in detail, the effective strain and void volume fraction of 5 elements nearby the fracture location are extracted as shown in Fig 8. From Fig 9, we can know that the void distribution of these elemets relatively homogeneous before $0.04 \mathrm{~s}$, but after that, the void fraction of these elemets begin to separate while the growth rate of element 1 is larger than others. Then, the void volume fraction nearly keeps constant after necking happened. The same rule can be summaried from the evolution of these elements' strain.

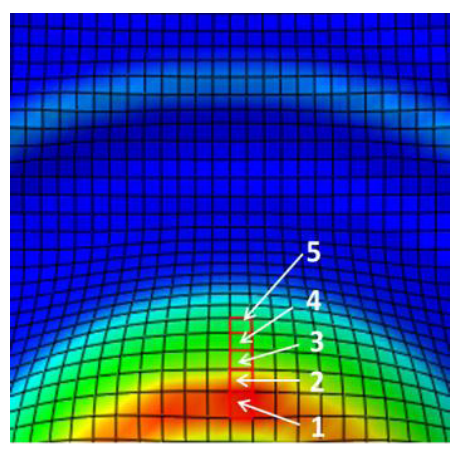

Figure 8. Extraction of analytical elements

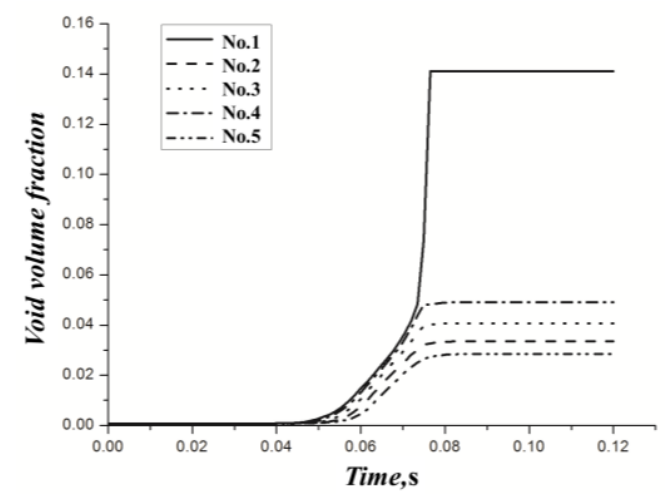

(a) Void damage evolution of 5 elements

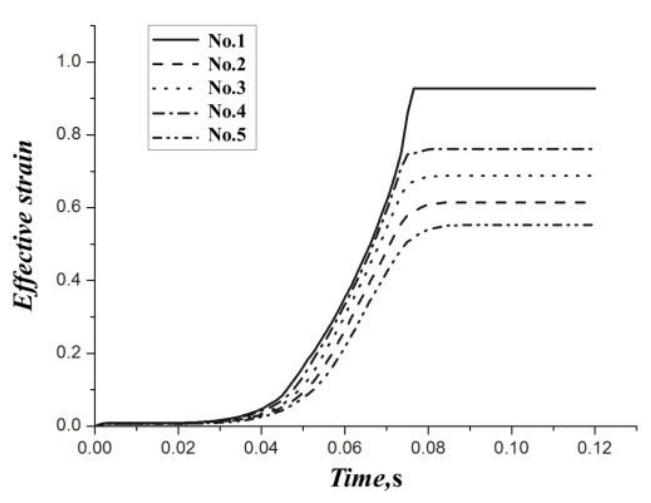

(b) Effective strain evolution of 5 elements

Figure 9. Comparison of void fraction and effective strain for different elements

\section{Conclusions}

The GTN damage model is used to research the damage evolution of high stength steel $22 \mathrm{MnB} 5$ at elevated temperature. A hybrid method integrated experiment and numerical technique is employed to identify the damage parameters. The results show that the damage parameters are different with the variation of temperature. This may be explained by the variation of $22 \mathrm{MnB} 5$ microstructure and further investigation like SEM analysis should be done to provide more detail state. The bugling forming at a deformation temperature of $700^{\circ} \mathrm{C}$ has been conducted as well as the corresponding simulation. The compraison between experimental and numerical results show the availability of GTN damage model in analyzing sheet formability at elevated temperature condition.

\section{Acknowledgement}

This work was funded by the Key Project of the National Natural Science Foundation of China (No.11272075) and China Postdoctoral Science Foundation (2014M561223) and the Fundamental Research Funds for the Central Universities (DUT15TD31)

\section{References}

1. A. Mecke, I. Lee, J.R. Baker jr., M.M. Banaszak Holl, B.G. Orr, Eur. Phys. J. E 14, 7 (2004)

2. 1. H. Karbasian, A.E. Tekkaya, J MATER PROCESS TECH, 210, 15 (2010) 2103-2118.

3. 2. F.A. McClintock, Journal of applied mechanics, 35, 2 (1968) 363-371.

4. 3. A. L. Gurson, Journal of Engineering Materials and Technology-Transactions of the ASME, 99 (1977) 2-15.

5. 4. H. Klöcker, V. Tvergaard, INT J FRACTURE, 106, 3 (2000) 259-276.

6. 5. H. Tinet, H. Klöcker, J. Le Coze, ACTA MATER, 52, 13 (2004) 3825-3842.

7. 6. V. Tvergaard, A. Needleman, Acta Metallurgica, 32, 1 (1984) 157-169.

8. 7. V. Tvergaard, INT J FRACTURE, 18, 4 (1982) 237-252.

9. 8. C.C. Chu, A. Needleman, Journal of Engineering Materials and Technology, 102, 3 (1980) 249-256.

10. 9. I.I. CUESTA, J.M. ALEGRE, R. LACALLE, FATIGUE FRACT ENG M, 33, 11 (2010) 703-713. 